\title{
Características produtivas e reprodutivas de vacas Holandesas e mestiças Holandês x Gir no Planalto Central
}

\author{
Concepta McManus ${ }^{1}$, Rodrigo de Almeida Teixeira ${ }^{2}$, Laila Talarico Dias ${ }^{3}$, Helder \\ Louvandini $^{1}$, Eliandra Maria Bianchini Oliveira ${ }^{1}$
}

\author{
1 Universidade de Brasília, Campus Darcy Ribeiro, Caixa Postal: 04508, CEP: 70910-970, Brasília - DF. \\ 2 Universidade Estadual do Oeste do Paraná - Campus Mal. Cândido Rondon, CCA - Depto Zootecnia, CEP: 85960-000, Marechal Cândido \\ Rondon - PR. \\ 3 Universidade Estadual de Ponta Grossa - Campus Uvaranas, Depto Zootecnia, bloco F, sala 6, CEP: 84030-900, Ponta Grossa - PR
}

RESUMO - Com o objetivo de comparar o desempenho leiteiro e reprodutivo de vacas da raça Holandesa e mestiças Holandês $\times$ Gir na região do Planalto Central foram utilizados dados de 1.456 vacas de cinco composições raciais, originadas das raças Holandesa $(\mathrm{H})$ e Gir $(\mathrm{G})$ : puras $\mathrm{H} ; 3 / 4 \mathrm{H} 1 / 4 \mathrm{G} ; 1 / 2 \mathrm{H} 1 / 2 \mathrm{G} ; 3 /{ }_{8} \mathrm{H} 5 / 8 \mathrm{G}$; e $1 / 4 \mathrm{H} 3 / 4 \mathrm{G}$. Analisaram-se as características produção média diária de leite (PMD), número de dias em lactação (DL) e produção total da lactação corrigida para 305 dias (P305), idade ao primeiro parto (IPP), período de gestação (PG) e intervalo de partos (IEP). Os maiores valores de PMD e P305 foram obtidos para os animais $3 / 4 \mathrm{H} 1 / 4 \mathrm{G}$ e $1 / 2 \mathrm{H} 1 / 2 \mathrm{G}$ e os menores, para os animais com maior proporção da raça Gir (1/44 $3 / 4 \mathrm{G})$. Os valores das características reprodutivas IPP e IEP, no entanto, foram menores para os animais $3 / 4 \mathrm{H} 1 / 4 \mathrm{G}$. Esses resultados evidenciam a adaptação das vacas mestiças ao ambiente a que foram submetidas ou que os animais puros da raça Holandesa, em razão do estresse nutricional e/ou térmico, não expressaram todo o seu potencial genético para produção leiteira. Além disso, os dados comprovaram a importância da utilização de sistemas de cruzamento na manutenção da produção dos animais e na sua adaptação ao ambiente.

Palavras-chave: cerrado, cruzamento, lactação

\section{Production and reproduction traits in Holstein and Gyr crossbred cows in the Central Plateau, Brazil}

\begin{abstract}
Data from 1456 purebred and crossbred cows of five different crossbred groups involving the Holstein $(\mathrm{H})$ and $\mathrm{Gyr}(\mathrm{G})$ breeds $\left(\mathrm{H} ; 3 / 4 \mathrm{H} 1 / 4 \mathrm{G} ; 1 / 2 \mathrm{H} 1 / 2 \mathrm{G} ; 3 / 8 \mathrm{H}^{5 / 8} \mathrm{G}\right.$ e $\left.1 / 4 \mathrm{H} 3 / 4 \mathrm{G}\right)$ were used to compare their milk production and reproductive traits in the Brazilian central plateau region. The performance traits studied were: average daily milk production (DMP); lactation length (LL); total production in 305 days (P305) and age at first calving (AFC), gestation length (GL) and calving interval (CI). Higher averages of DMP and P305 were observed for animals $3 / 4 \mathrm{H} 1 / 4 \mathrm{G}$ and $1 / 2 \mathrm{H} 1 / 2 \mathrm{G}$ and the smallest ones for crossbred animals with larger proportion of the Gyr breed $(1 / 4 \mathrm{H} 3 / 4 \mathrm{G})$. The lowest values of AFC and CI were observed for $3 / 4 \mathrm{H} 1 / 4 \mathrm{G}$ animals. These results indicate the importance of crossbreeding strategies to produce animals that are more adapted to the environmental conditions of the region. Holstein purebreds probably do not express their full genetic potential due to nutritional and/or heat stress.
\end{abstract}

Key Words: Brazilian central plateau, crossbreeding, lactation

\section{Introdução}

A viabilidade econômica da produção leiteira nacional está relacionada à escala de produção, na qual grandes produtores obtêm insumos a preços melhores, reduzindo os custos e possibilitando a oferta do produto em melhores condições, o que aumenta as margens de lucro. A eficiência de produção tem influência direta na rentabilidade do agronegócio leiteiro no Brasil (Ferreira \& Madalena, 1997). Atualmente, a maior parte da produção de leite é prove- niente de animais mestiços zebuínos criados em pastagens, com pouca tecnologia e baixo custo de produção (Freitas et al., 2001; Facó et al., 2002).

O Cerrado brasileiro não possui a tradição leiteira de alguns estados da Região Sudeste, porém, como ocorre no estado de Goiás, a pecuária leiteira pode ser melhorada e passar a ser uma importante alternativa de renda aos produtores rurais do Distrito Federal. Parte dos pecuaristas dessa região acredita que rebanhos com maior proporção da raça holandesa são ideais para produção de leite, no entanto, a

Este artigo foi recebido em 22/5/2006 e aprovado em 30/10/2007.

Correspondências devem ser enviadas para concepta@unb.br. 
eficiência produtiva e reprodutiva dos animais está relacionada à adaptação de seus genótipos ao conjunto de fatores ambientais que caracterizam o sistema de produção, favoráveis a alguns genótipos e desfavoráveis a outros. Portanto, para viabilizar o aumento da produção leiteira no Planalto Central, é importante identificar os genótipos adaptados à região.

A fim de contribuir nesse sentido, objetivou-se com este trabalho avaliar o desempenho produtivo e reprodutivo de vacas leiteiras de diferentes composições raciais envolvendo cruzamentos entre as raças Holandesa e Gir na região do Planalto Central.

\section{Material e Métodos}

Foram utilizados registros de 1.456 parições de vacas leiteiras de cinco composições genéticas das raças Holandês

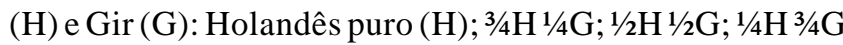
$\mathrm{e}^{3 / 8} \mathrm{H} 5 / 8 \mathrm{G}$. As informações foram obtidas entre 1993 e 2002 em rebanho de uma fazenda localizada no estado de Goiás, na divisa com o Distrito Federal, com características de manejo nutricional e sanitário semelhantes às das demais propriedades da região. Os animais foram criados em pastagem (Brachiaria decumbens, Brachiaria ruziziensis, Brachiaria brizantha cv. Marandu, e Andropogon gayanus cv. Planaltina), porém, durante a estação seca, utilizaram-se silagem de milho e sal mineral à vontade na alimentação dos animais e concentrado para as vacas em lactação. O controle leiteiro foi baseado em duas ordenhas diárias e as informações da produção leiteira foram coletadas a cada 14 dias.

As características analisadas foram produção média de leite (PMD kg/dia); dias em lactação (DL); produção total da lactação corrigida para 305 dias (P305), com 1.456 informações; período de gestação (PG), com 660 registros; intervalo de partos (IEP), com 1.010 registros; e idade ao primeiro parto (IPP), com 370 informações.

As análises estatísticas foram realizadas utilizando-se o método dos quadrados mínimos, por meio do procedimento GLM do software SAS (SAS, 1999).

Para todas as características analisadas, o modelo estatístico utilizado incluiu grupo genético, mês e ano de parição e ordem do parto (exceto para idade ao primeiro parto). No estudo das variáveis PMD, P305 e IEP, consideraram-se como co-variáveis no modelo os efeitos de dias em lactação(efeitos linear e quadrático) e, na análise de DL, incluíram-se os efeitos linear e quadrático da produção total de leite. Os modelos podem ser representados por: $y_{i j k l m n}=\mu+G G_{i}+M_{j}+A_{k}+O_{m}+b_{1}\left(D L_{i j k m n}-\overline{D L}\right)+b_{2}\left(D L_{j k l m n}-\overline{D L}\right)^{2}+e_{i j k l m n}$ $y_{i j k m n}=\mu+G G_{i}+M_{j}+A_{k}+O_{m}+b_{1}\left(P 305_{i j k m n}-\overline{P 305}\right)+b_{2}\left(P 305_{j k k m n}-\overline{P 305}\right)^{2}+e_{i j k m n}$ em que: $\mathrm{y}_{\mathrm{ijklm}}=$ valor observado para variáveis dependentes; $\mathrm{m}=$ intercepto; $\mathrm{GG}_{\mathrm{i}}=$ efeito do iésimo grupo genético; $\mathrm{M}_{\mathrm{j}}=$ efeito do jésimo mês de parto; $A_{k}=$ efeito do $\mathrm{k}^{\text {ésimo }}$ ano de parto; $\mathrm{O}_{\mathrm{m}}=$ efeito da $\mathrm{m}^{\text {ésima }}$ ordem de parto; $\mathrm{DL}{ }_{\mathrm{ijklm}}=$ dias em lactação; DL = média de dias em lactação; P305 ${ }_{\text {ijklmn }}=$ produção total de leite ajustada para 305 dias; P305 = média de produção total de leite ajustada para 305 dias; $b_{1}$ e $b_{2}=$ coeficiente de regressão linear e quadrático para a co-variável incluída em cada modelo, respectivamente; $\mathrm{e}_{\mathrm{ijklmn}}=$ erro aleatório associado a cada observação.

\section{Resultados e Discussão}

O efeito de grupo genético foi estatisticamente significativo $(\mathrm{P}<0,10)$ para todas as características estudadas (Tabela 1), no entanto, o efeito de mês de parição não influenciou $(\mathrm{P}>0,10)$ nenhuma das características avaliadas. As vacas com menores ordens de parto foram menos influenciadas pelo estresse das altas temperaturas do verão em comparação àquelas com maiores ordens de parto. Tendência semelhante foi relatada por Durães \& Keown (1991), em estudo com dados de rebanhos leiteiros de nove estados localizados na região meio-oeste dos Estados Unidos.

Os efeitos de ano de nascimento e de ordem de parição foram significativos $(\mathrm{P}<0,01)$ para as características $\mathrm{PMD}$, P305 e IEP, indicando a importância de se considerar os fatores ambientais em estudos sobre a eficiência produtiva. No entanto, apenas para PMD os efeitos linear e quadrático da co-variável DL foram significativos $(\mathrm{P}<0,10)$. Os efeitos de ano, ordem de parto e da co-variável P305 (linear e quadrático) tiveram influência $(\mathrm{P}<0,05)$ sobre a variável dias em lactação.

Com exceção do efeito de grupo genético, nenhuma variável incluída no modelo apresentou efeito significativo sobre a PG. O efeito de ano de parição, no entanto, influenciou $(\mathrm{P}<0,01)$ a IPP.

Tanto os valores da produção diária de leite (PMD) quanto os da produção em 305 dias (P305) aumentaram conforme a ordem de lactação e tiveram pico na $6^{\mathrm{a}}$ lactação; porém, nas lactações subseqüentes, as produções foram inferiores. Os valores variaram entre 11,17 e 7,38 kg/dia e $3.245,18$ e 2.292,20 kg para PMD e P305, respectivamente. Resultado semelhante foi obtido por Lopes et al. (1996), que destacaram que vacas próximas à maturidade fisiológica tiveram produção leiteira superior.

Quando analisado o efeito da ordem de lactação sobre a característica dias em lactação (DL), verificou-se que os 
Tabela 1 - Resumo da análise de variância para as características estudadas

\begin{tabular}{|c|c|c|c|c|c|c|}
\hline & PMD (kg/dia) & DL (dias) & P305 (kg) & PG (dias) & IEP (dias) & IPP (dias) \\
\hline Grupo genético & $* * *$ & $* *$ & $* * *$ & $*$ & $* * *$ & $* * *$ \\
\hline Mês & $\mathrm{ns}$ & $\mathrm{ns}$ & $\mathrm{ns}$ & $\mathrm{ns}$ & $\mathrm{ns}$ & $\mathrm{ns}$ \\
\hline Ano & $* * *$ & $* * *$ & $* * *$ & $\mathrm{~ns}$ & $*$ & $* * *$ \\
\hline Ordem de parto & $* * *$ & $* *$ & $* * *$ & $\mathrm{~ns}$ & $* * *$ & \\
\hline DL & $*$ & & $\mathrm{~ns}$ & & $\mathrm{~ns}$ & \\
\hline $\mathrm{DL}^{2}$ & * & & $\mathrm{ns}$ & & $\mathrm{ns}$ & \\
\hline P305 & & $* * *$ & & & & \\
\hline $\mathrm{P} 305^{2}$ & & $* *$ & & & & \\
\hline $\mathrm{R}^{2}$ & 0,74 & 0,17 & 0,81 & 0,17 & 0,27 & 0,57 \\
\hline $\mathrm{CV}$ & 19,68 & 13,40 & 23,04 & 16,97 & 19,35 & 12,98 \\
\hline Média & 9,69 & 283,79 & $2.865,17$ & 285,38 & 424,17 & $1.209,16$ \\
\hline
\end{tabular}

*** $\mathrm{P}<0,01 ;{ }^{* *} \mathrm{P}<0,05 ;{ }^{*} \mathrm{P}<0,10 ;$ ns - não-significativo; $\mathrm{PMD}=$ produção média diária; $\mathrm{DL}=$ duração da lactação; $\mathrm{P} 305=$ produção em 305 dias; $P G=$ período de gestaçấ; $\mathrm{IEP}=$ intervalo de partos; IPP = idade ao primeiro parto.
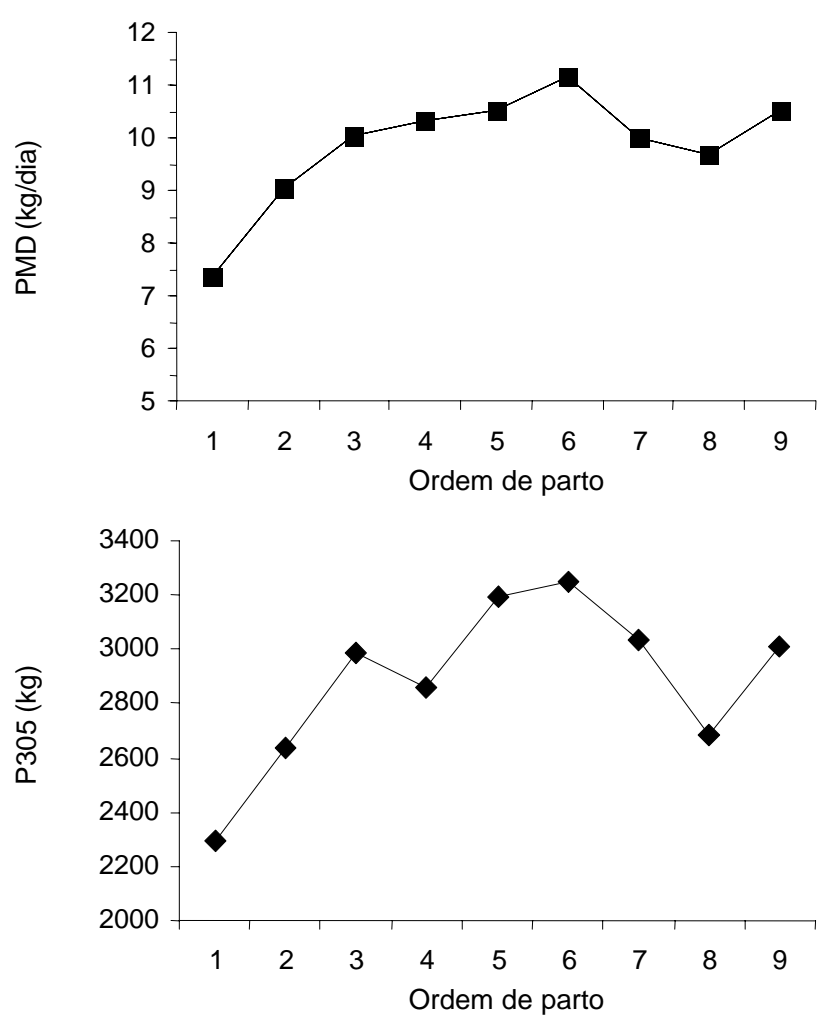

Figura 1 - Ordem de parição sobre a produção diária de leite (PMD) e a produção em 305 dias (P305).

maiores períodos de DL ocorreram nas vacas mais jovens, o que está de acordo com o relatado por Campos (1987), que sugeriu que esse efeito seria decorrente do maior período de serviço dos animais jovens.

Observou-se influência da composição racial sobre os valores de PMD e P305, que foram maiores nos animais $3 / 4 \mathrm{H}^{1 / 4} \mathrm{G} \mathrm{e}^{1 / 2} \mathrm{H}^{1 / 2} \mathrm{G}$ e menores nos animais com maior proporção da raça Gir $(1 / 4 \mathrm{H} 3 / 4 \mathrm{G})$ (Tabela 2). Resultados semelhantes foram relatados por Guimarães et al. (2002) e Facó et al.
(2002), que observaram maior produção de leite por lactação para os animais $7 / 8{ }_{8}{ }_{1} \mathrm{G}$ e $3 / 4 \mathrm{H} 1 / 4 \mathrm{G}$. O PMD das vacas Holandesas puras foi inferior ao do grupo de animais ${ }_{5} / \mathrm{G}_{3}^{3} / 8 \mathrm{H}$, o que evidencia a adaptação das vacas mestiças, principalmente as $1 / 2 \mathrm{H}^{1 / 2} \mathrm{G}$ e $3 / 4 \mathrm{H}^{1} / 4 \mathrm{G}$, ao ambiente a que foram submetidas ou que, nessas condições de criação, em razão do estresse nutricional e/ou térmico, provavelmente os animais puros da raça Holandesa não expressaram todo o seu potencial genético para produção leiteira. O termo adaptação nesse caso deve ser compreendido em sentido amplo e significa que os tipos de ação gênica atuantes nos genótipos dos animais mestiços propiciaram o desempenho desses animais.

Os animais com maior proporção da raça $\mathrm{Gir}(1 / 4 \mathrm{H} 3 / 4 \mathrm{Ge}$ $3 / 8 \mathrm{H}_{8}^{5 / 8} \mathrm{G}$ ) apresentaram maior duração da lactação (DL) e não diferiram estatisticamente entre si. A duração das lactações dos animais puros $(\mathrm{H}), 1 / 2 \mathrm{H}^{1 / 2} \mathrm{Ge}^{3 / 8} \mathrm{H}_{8}^{5 / 8} \mathrm{G}$ foram semelhantes entre si, mas as lactações mais curtas foram observadas nos animais $3 / 4 \mathrm{H} 1 / 4 \mathrm{G}$.

Estes resultados diferem dos obtidos na região de Viçosa, Minas Gerais, por Campos (1987), que relatou aumento da duração da lactação conforme aumentou a proporção da raça Holandesa. Os resultados indicam, no entanto, a mesma tendência observada para as características PMD e P305, em que o ambiente provavelmente foi fator limitante à produção de leite das vacas puras. Segundo Faria \& Silva (1996), a duração ideal da lactação é de dez meses, índice obtido em animais com maior proporção racial da raça Holandesa, porém, possivelmente em virtude das diferenças ambientais, este resultado não foi observado nas condições do Planalto Central para esse raça.

Os resultados obtidos para o período de gestação indicam que vacas com maior composição da raça Holandesa tiveram gestações mais longas em comparação àquelas com maior proporção da raça Gir, o que provavelmente está 
Tabela 2 - Médias e erros-padrão estimados por quadrados mínimos para as características analisadas em cada grupo genético

\begin{tabular}{lccccc}
\hline & \multicolumn{5}{c}{ Grupo genético $(\mathrm{GG})$} \\
\cline { 2 - 6 } & $\mathrm{H}$ & $3 / 4 \mathrm{H} 1 / 4 \mathrm{G}$ & $1 / 2 \mathrm{H} 1 / 2 \mathrm{G}$ & $3 /{ }_{8} \mathrm{H} 5 / 8 \mathrm{G}$ & $1 / 4 \mathrm{H} 3 / 4 \mathrm{G}$ \\
\hline $\mathrm{n}$ & 138 & 77 & 65 & 936 & 240 \\
$\mathrm{PMD}$ & $8,8 \mathrm{c} \pm 3,0$ & $12,6 \mathrm{a} \pm 3,6$ & $11,5 \mathrm{a} \pm 3,1$ & $9,5 \mathrm{~b} \pm 2,1$ & $7,9 \mathrm{~d} \pm 3,1$ \\
P305 & $3.049,2 \mathrm{~b} \pm 1.191,8$ & $3.823,3 \mathrm{a} \pm 1.170,3$ & $3.473,4 \mathrm{a} \pm 1.178,4$ & $2.759,5 \mathrm{bc} \pm 1.131,5$ & $2.348,8 \mathrm{c} \pm 1.058,2$ \\
DL & $279,6 \mathrm{~b} \pm 28,0$ & $274,4 \mathrm{c} \pm 7,2$ & $279,3 \mathrm{~b} \pm 27,3$ & $281,5 \mathrm{ab} \pm 29,6$ & $284,4 \mathrm{a} \pm 36,0$ \\
PG & $311,1 \mathrm{a} \pm 9,6$ & $300,7 \mathrm{a} \pm 6,4$ & $299,9 \mathrm{a} \pm 5,2$ & $275,4 \mathrm{~b} \pm 4,9$ & $277,6 \mathrm{~b} \pm 4,1$ \\
IEP & $458,0 \mathrm{a} \pm 17,2$ & $355,7 \mathrm{c} \pm 15,7$ & $442,9 \mathrm{a} \pm 19,1$ & $412,0 \mathrm{~b} \pm 17,2$ & $438,8 \mathrm{ab} \pm 20,1$ \\
IPP & $1.069,0 \mathrm{~b} \pm 117,2$ & $844,4 \mathrm{c} \pm 91,3$ & $1.003,1 \mathrm{~b} \pm 97,1$ & $1.240,2 \mathrm{a} \pm 123,2$ & $1.278,8 \mathrm{a} \pm 120,1$ \\
\hline
\end{tabular}

Os códigos dos $G G$ representam a proporção das raças Holandês $(H)$ e $\operatorname{Gir}(G) ; n=$ observaçães; $P M D=$ produção média diária; $D L=$ duração da lactação P305 = produção em 305 dias; $P G$ = período de gestação; IEP = intervalo de partos; IPP = idade ao primeiro parto. Médias seguidas de letras iguais nas linhas não diferem entre si pelo teste Tukey $(P<0,05)$.

relacionado ao peso ao nascer dos bezerros. De acordo com Valle (1995), animais da raça Holandesa, com maior porte, são mais pesados ao nascer, assim, as vacas podem apresentar maiores períodos de gestação.

Os valores obtidos para o intervalo de partos (IEP) foram menores entre os animais $3 / 4 \mathrm{H} 1 / 4 \mathrm{G}$ e os maiores entre os animais $1 / 2 \mathrm{H}^{1 / 2} \mathrm{G}$ e $1 / 4 \mathrm{H} 3 / 4 \mathrm{G}$. Lemos et al. (1992) relataram que animais $\mathrm{F}_{1}$ foram superiores em fazendas de nível baixo de manejo, enquanto os $5 /{ }_{8} \mathrm{H} \mathrm{3/}{ }_{8} \mathrm{G}$ foram melhores nas fazendas de nível alto de manejo.

Em estudo realizado por Madalena et al. (1990), animais $\mathrm{F}_{1}$ apresentaram maior vigor do híbrido, traduzido em maior precocidade e peso ao início da função sexual. A heterose para produção de leite é importante em cruzamentos entre raças européias e zebuínas e apresenta valores médios que variam de 28\% (Cunningham \& Syrstad, 1987) a 17,3\% (Rege, 1998). Para características reprodutivas e adaptativas, a heterose tem grande importância e apresenta valores médios de 5,8\% para duração da lactação, $11 \%$ para idade ao primeiro parto e $9 \%$ para intervalo de partos, entre outras características (Rege,1998). No entanto, entre raças européias, a heterose para produção de leite é relativamente baixa e próxima a 5\% (Touchberry, 1992), contudo, os valores de heterose obtidos em regiões tropicais podem ser subestimados, em virtude da freqüente eliminação nas análises de dados considerados "anormais", principalmente os de lactações curtas, procedimento que reduz a variação genética entre os grupos de cruzamentos (Madalena et al., 1992).

Além das diferenças genéticas entre os animais, uma das hipóteses para explicar os valores elevados de IEP é que alguns produtores, por conveniência, retardam a inseminação de animais que atingem altos níveis de produção no início do período de lactação (Grossi \& Freitas, 2002).

Os resultados obtidos para idade ao primeiro parto (IPP) comprovam que os animais $3 / 4 \mathrm{H} 1 / 4 \mathrm{G}$ foram os mais precoces, indicando que os animais desta composição racial foram reprodutivamente mais eficientes e provavelmente tiveram maior produção de bezerros em mesmo intervalo de tempo. As vacas Holandesas e $1 / 2 \mathrm{H}^{1 / 2} \mathrm{G}$ apresentaram IPP estatisticamente iguais e com valores inferiores aos obtidos para os animais $3 / 4 \mathrm{H} 1 / 4 \mathrm{G}$. Entretanto, os animais mais tardios foram aqueles com maiores proporções da raça Gir: ${ }_{8} / \mathrm{H}_{5}^{5 / 8} \mathrm{G}$ e $1 / 4 \mathrm{H} 3 / 4 \mathrm{G}$.

Como as características reprodutivas são fortemente influenciadas por efeitos ambientais, neste estudo, as diferenças entre os valores de IPP podem ter sido causadas por uma prática comum a alguns criadores que pré-determinam a idade e o peso para que as novilhas sejam expostas pela primeira vez à reprodução, o que aumentaria a idade à primeira cria.

\section{Conclusões}

A exploração de sistemas de cruzamento pode beneficiar o desempenho produtivo e reprodutivo da atividade leiteira, pois os melhores desempenhos das características produtivas foram obtidos com animais $1 / 2 \mathrm{H}^{1 / 2} \mathrm{G}$ e $3 / 4 \mathrm{H} 1 / 4 \mathrm{G}$. Animais com maior proporção da raça Gir apresentaram maiores durações da lactação, porém, a maior duração da lactação não foi suficiente para superar a produção média diária de leite e a produção total da lactação corrigida para 305 dias dos animais com maior composição da raça Holandesa. Os melhores resultados de características reprodutivas foram obtidos com a composição racial $3 / 4 \mathrm{H}^{1 / 4} \mathrm{G}$. As condições ambientais foram limitantes para animais puros da raça Holandesa expressarem seu potencial genético para as características analisadas.

\section{Agradecimento}

Ao CNPq, pela bolsa de produtividade concedida à primeira autora. 


\section{Literatura Citada}

CAMPOS, J.M.S. Aspectos reprodutivos e produtivos em um sistema de produção de leite, na microrregião de Viçosa, Estado de Minas Gerais. Viçosa, MG: Universidade Federal de Viçosa, 1987. 109p. Dissertação (Mestrado em Zootecnia) Universidade Federal de Viçosa, 1987.

CUNNINGHAM, E.P.; SYRSTAD, O. Crossbreding Bos indicus and Bos taurus for milk production in the tropics. Rome: FAO, 1987. 90p

DURÃES, M.C.; KEOWN, J.F. Age-month factors. Mature equivalent factors for three yield traits for non-registered and registered cattle. Revista Brasileira de Genética, v.14, p.713$728,1991$.

FACÓ, O.; LÔBO, R.N.B.; MARTINS FILHO, R. et al. Análise do desempenho produtivo de diversos grupos genéticos Holandês $\mathrm{x}$ Gir no Brasil. Revista Brasileira de Zootecnia, v.31, n.5, p.1944-1952, 2002 .

FARIA, V.P.; SILVA, S.C. Fatores biológicos determinantes na pecuária leiteira. In: SIMPÓSIO INTERNACIONAL - O FUTURO DOS SISTEMAS DE PRODUÇÃO DE LEITE, 1996, Juiz de Fora. Anais... Coronel Pacheco: CNPGL/EMBRAPA, 1996. p.77-89.

FERREIRA, J.J.; MADALENA, F.E. Efeito do sistema de cruzamento sobre o desempenho produtivo e reprodutivo de vacas leiteira. Arquivo Brasileiro de Medicina Veterinária e Zootecnia, v.49, n.6, p.74-75, 1997.

FREITAS, M.S.; DURÃES, M.C.; FREITAS, A.F. et al. Comparação da produção de leite e de gordura e da duração da lactação entre cinco "graus de sangue" originados de cruzamentos entre Holandês e Gir em Minas Gerais. Arquivo Brasileiro de Medicina Veterinária e Zootecnia, v.53, n.6, p.708-713, 2001.

GROSSI, S.F.; FREITAS, M.A.R. Eficiência reprodutiva e produtiva em rebanhos leiteiros comerciais monitorados por sistema informatizado. Revista Brasileira de Zootecnia, v.31, n.3, p.1362-1366, 2002.

GUIMARÃES, J.D.; ALVES, N.G.; COSTA, E.P. et al. Eficiências reprodutiva e produtiva em vacas das raças Gir, Holandês e cruzadas Holandês x Zebu. Revista Brasileira de Zootecnia, v.31, n.2, p.641-647, 2002.

LEMOS, A.M.; MADALENA, F.E.; TEODORO, R.L. et al. Comparative performance of six Holstein-Friesian x Guzera grades in Brazil. 5. Age at first calving. Revista Brasileira de Genética, v.15, n.1, p.73-83, 1992.

LOPES, M.A.; NEIVA, R.S.; VALENTE, J. et al. Aplicação da função tipo gama incompleta no estudo da curva de lactação de vacas da raça Holandesa, variedade preta e branca, mantidas em sistema intensivo de produção. Revista Brasileira de Zootecnia, v.25, n.6, p.1086-1111, 1996.

MADALENA, F.E.; LEMOS, A.M.; TEODORO, R.L. et al. Dairy production and reproduction in Holstein-Friesian and Guzera crosses. Journal of Dairy Science, v.73, n.7, p.1872-1886, 1990.

MADALENA, F.E.; LEMOS, A.M.; TEODORO, R.L. Consequences of removing the variation in lactation length on the evaluation of dairy cattle breeds and crosses. Brazilian Journal of Genetics, v.15, n.3, p.585-593, 1992.

REGE, J.E.O. Utilization of exotic germplasm for milk production in the tropics. In: WORLD CONGRESS ON GENETICS APPLIED TO LIVESTOCK PRODUCTION, 6., 1998, Armidale. Proceedings... Armidale, 1998. v.25, p.193-200.

STATISTICAL ANALYSIS SYSTEM - SAS. User's guide: statistics. Cary: SAS Institute, 1999. (CD-ROM).

TOUCHBERRY, R.W. Crossbreeding effects in dairy cattle: the Illinois experiment, 1949 to 1969 . Journal of Dairy Science, v.75, p.640-667, 1992.

VALLE, A. Duración de gestación, producción de leche e intervalo entre partos de vacas Holstein de distintas procedencias. Zootecnia Tropical, v.13, n.2, p.199-214, 1995. 